\title{
Characterization of vpr Vector Constructed from Chimeric Simian and Human Immunodeficiency Virus
}

\author{
Takeshi HAGA $^{1) *}$, Masashi OKOBA ${ }^{1)}$, Nanase YAMAZAKI ${ }^{1)}$, Shino KUMABE ${ }^{1)}$, Yuya SHIMIZU ${ }^{1)}$, Yoshitaka GOTO ${ }^{1)}$, \\ Takeo KUWATA ${ }^{2)}$, Iouri L. KOZYREV ${ }^{2)}$, Masanori HAYAMI ${ }^{2)}$ and Tomoyuki MIURA ${ }^{2)}$ \\ ${ }^{1)}$ Department of Veterinary Microbiology, Miyazaki University, 1-1 Gakuen Kibanadai Nishi, Miyazaki 889-2192 and ${ }^{21}$ Laboratory of \\ Viral Pathogenesis, Institute for Virus Research, Kyoto University, 53 Shogoin-Kawahara-cho, Sakyo-Ku, Kyoto 606-8507, Japan
}

(Received 1 October 2002/Accepted 17 January 2003)

ABSTRACT. Chimeric simian and human immunodeficiency viruses (SHIVs) are useful tool for investigating AIDS pathogenesis and for development of vaccine. We constructed a SHIV-vpr vector (designated as SHIV-3sj) by replacing $v p r$ region with restriction enzyme sites. SHIV-3sj was designed to express inserted gene along with its viral replication. Five cytokine genes were inserted into SHIV$3 \mathrm{sj}$, and ability of viral replication and expression of the inserted genes were examined. The short insert including RANTES and IL-5 resulted in the successful expression from SHIV-3sj, while the construct having longer genes including IL-2, IL-6 and IL-12p35 failed to become replication competent. These results suggest that the length of the insert is an important factor for the replication ability of SHIV-3sj vector.

KEY WORDS: SHIV, vector, $v p r$.

J. Vet. Med. Sci. 65(5): 633-636, 2003

In the research of human immunodeficiency virus type-1 (HIV-1), the lack of a suitable animal model is a major obstacle, because few animal species are susceptible to HIV-1 infection. Chimeric simian and human immunodeficiency viruses (SHIVs) that encode HIV-1 Env and are infectious to macaques have been utilized to study the pathogenicity of HIV-1 in vivo. In addition, SHIVs are candidates for a vaccine against HIV-1 because attenuated SHIVs can induce long-lasting anti-HIV-1 humoral and cell-mediated immunity in monkeys without AIDS-like diseases [3].

To add an adjuvant effect for SHIV as vaccine and to study the role of cytokines in AIDS pathogenesis, we sought to develop SHIV vectors. SHIV vectors are designed to express inserted gene (such as cytokines) along with the viral replication. We first constructed SHIV-nef vector, and successful expression was confirmed in previous studies $[2,4,7,9]$. However, nef has many important function, and early study on SIV-macaque model indicated that nef is required for development of AIDS [6]. To reflect the function of nef, construction of other vector is required.

$V p r$ and $v p x$ are related genes, and it has been suggested that the vpx gene arose by the duplication of an ancestral $v p r$ [12]. The results from macaques infected with mutant SIV showed that progression to AIDS can occur in the absence of a gene for $v p r$, but $v p r+v p x$ double mutant resulted in the decreased virulence which appears to be similar in severity to that of the nef deletion mutant [1]. Therefore we constructed SHIV-vpr vector designated as SHIV-3sj, which has a gene for $v p x$ and deletion of a gene for $v p r$. As we reported, expression of interleukin (IL)-5 was succeeded from SHIV-3sj [7]. In this paper, we describe the characteristics of SHIV-3sj.

SHIV NM-3rN was chosen as the SHIV backbone for the

\footnotetext{
* Correspondence to: Haga, T., Department of Veterinary Microbiology, Miyazaki University, Miyazaki 889-2192, Japan.
}

$v p r$ vector construct, because of its growth advantage in macaque monkeys [8]. NM-3rN consists of $v p r, v p u, e n v$, tat and rev genes from HIV-1 NL432, and the other genes from SIV mac239. To generate SHIV-3sj vector, the sequence of SHIV NM-3rN $v p r$ was changed by two-step PCR. The sequence 5'-ACTAGT ATGAC TCTAGA GTCGC GCATGC-3' was introduced as indicated in Fig. 1a (underlined sequence shows Spe I, Xba I and Sph I restriction enzyme sites, respectively). To retain the splicing acceptor site for tat gene, the sequence from nucleotide number (nt) 5749 of NL432 (GenBank accession number M19921: 5'-TGCAACAA---) remains intact in SHIV-3sj vector. SHIV-3sj vector lacks $v p r$ open reading frame (ORF), but $v p x$ in this vector is thought to be functional because boxed TGA works as stop codon for $v p x$ (Fig. 1b).

To test the function of the vector, 5 cytokine genes were inserted into SHIV-3sj independently. These include human RANTES [10], IL-5, IL-2, IL-6 and $35 \mathrm{kDa}$ subunit of IL-12 (IL-12p35). For cloning of each gene, the sequence of 5'-GCTAGC ATAA-3' (Nhe I site is shown in italic, stop codon for $v p x$ gene is boxed) and 5'-GCATGC-3' (Sph I site) were added at the flanking region of the ORF for each gene. The gene was amplified with the modified primers by PCR, and cloned into pUC119 vector by TA-cloning. The sequence of amplified region was confirmed by sequencing with an ABI 310 sequencer (Applied Biosystems, Foster City, CA, U.S.A.). The PCR fragment digested with Nhe I and Sph I was then inserted into the SHIV-3sj vector at Spe I and $S p h$ I region. After the recombination, boxed TAA works as stop codon for $v p x$. As result, vpr gene was replaced with the ORF for inserted gene (Fig. 1b).

To observe the replication ability, the construct was transfected onto M8166 CD4 positive human lymphocyte cell line, which is often used for the SHIV experiments because of its high susceptibility to the virus. Ten microgram of the DNA was transfected with DEAE-dexrtan method as described previously [11]. The culture medium was 
a)

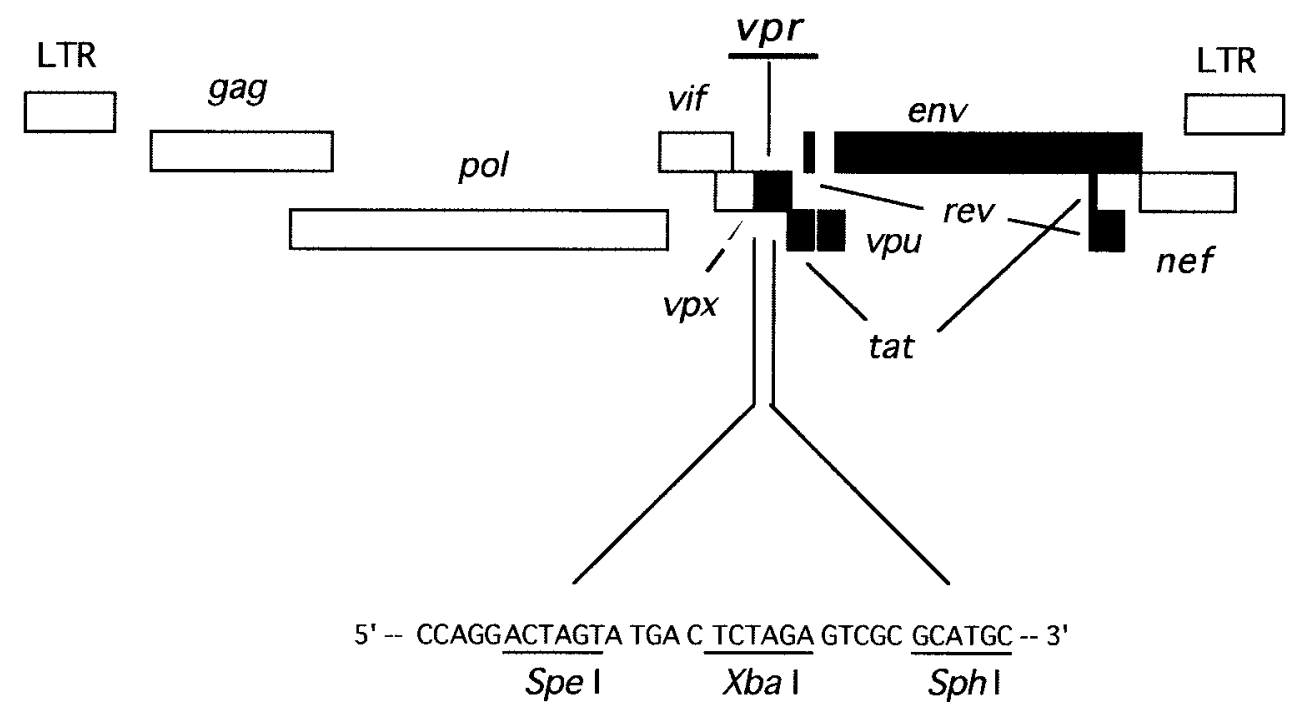

b)

(i) $\mathrm{NM}-3 \mathrm{rN}$

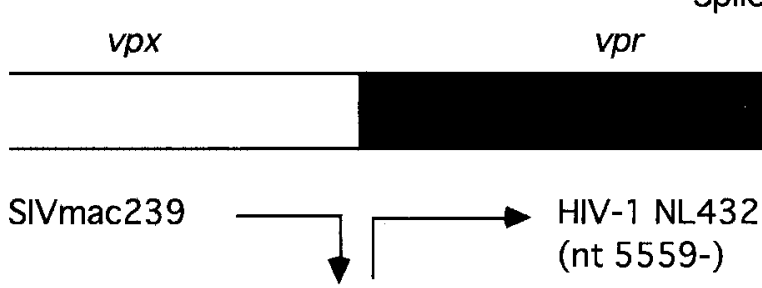

(nt 5749)

(nt 5559-)

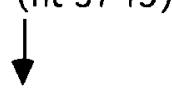

5' -- CCAGG ACT AGC A TAA ATG (ORF for VPR) TAA GAATTC TGC AACAA - 3

(ii) SHIV-3sj

5'-- CCAGg aCt AGt A TGA C TCTAGA GTCGC
Spel

(iii) flanking of ORF for RANTES (gene for insertion)

(iv) SHIV3sj-RANTES
$5_{1}-$

\author{
GCT AGC A TAA ATA (ORF for RANTES) TAG \\ Nhe I
}

$5^{\prime}$-- CCAGG ACT AGC A TAA ATG (ORF for RANTES) TAG
Splicing Acceptor for tat

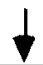

Fig. 1. Genetic structure of SHIV-3sj and insert. a) Genetic structure of SHIV-3sj. Black and white regions indicate the sequences derived from HIV-1NL432 (GenBank accession number: M19921) and SIVmac239 (GenBank accession number: M33262), respectively. $V p r$ region was replaced with the sequence as indicated including Spe I, Xba I and Sph I sites. b) Sequence around the junction region of SHIV-3sj vector and the insert. The boxed sequence indicates either start or stop codon for the open reading frame (ORF). The underlined sequence indicates ORF. Italic indicates restriction enzyme site as shown below the sequence. (i) NM-3rN: the p arental SHIV of SHIV-3sj vector. (ii) SHIV-3sj: $v p r$ vector described in this paper. (iii) flanking region of the ORF of the gene for insertion. In this figure, RANTES is shown as an example. (iv) SHIV3sj-RANTES: recombinant between SHIV-3sj and RANTES. RANTES ORF is inserted into the SHIV-3sj vector.

changed every 3 days, and the transfected cells were observed. Viral replication was monitored by virion-associated reverse transcriptase (RT) activity as described previously [13]. When infectious virus was recovered from the transfected cells, cell-free viral stock was made as described previously [2]. To investigate the kinetics of viral replication and expression of the inserted gene, M8166 cells were infected with the stock virus, and culture supernatant was 
Table 1. Summary of relationship among the genes inserted to SHIV-3sj, length of the open reading frame (ORF) for the insert, ability of viral replication, and expression of the gene

\begin{tabular}{lccc}
\hline Inserted Gene & Length of ORF & Viral Replication & Expression \\
\hline RANTES & $276 \mathrm{bp}$ & + & + \\
IL-5 & $399 \mathrm{bp}$ & $+^{\mathrm{a}}$ & $+^{\mathrm{a})}$ \\
IL-2 & $462 \mathrm{bp}$ & - & - \\
IL-6 & $639 \mathrm{bp}$ & - & - \\
IL-12 p35 & $762 \mathrm{bp}$ & - & - \\
\hline
\end{tabular}

a) reported in ref.7.

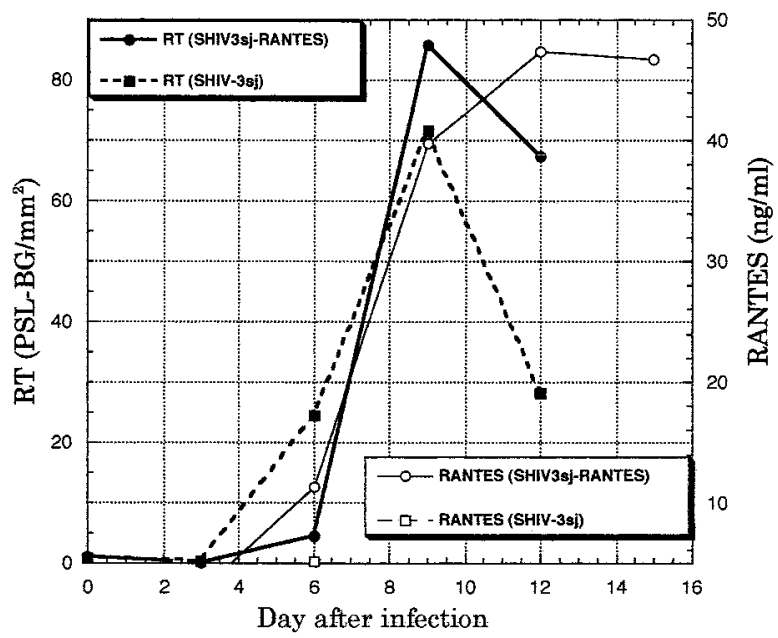

Fig. 2. Kinetics of virus replication and RANTES production of SHIV3sj-RANTES (-) and SHIV-3sj (- - ) in M8166 human CD4 positive $\mathrm{T}$ cell line. Viral replication was monitored by RT activity from the supernatant of SHIV3sj-RANTES (O) or SHIV-3sj ( $)$-infected cells. RANTES production was detected by ELISA from the supernatant of SHIV3sj-RANTES ( $\bigcirc$ ) or SHIV-3sj $(\square)$-infected cells. The unit for RT activity is PSL-BG, photostimulated luminescence minus background (FLA-3000, Fuji Film, Japan). The unit for RANTES $(n \mathrm{~g} / \mathrm{ml})$ was calculated on the basis of the standard provided in the ELISA kit (R\&D, Minneapolis, MN, U.S.A.).

collected every 3 days. Samples were subjected for RT assay and for ELISA as described previously [2].

Infectious virus was recovered from the cells transfected with SHIV3sj-RANTES, as well as SHIV3sj-IL5, which was previously reported [7]. However, in the cases of SHIV3sj-IL2, SHIV3sj-IL6 and SHIV3sj-IL12p35, neither cytopathic effect of the cells was observed nor RT activity in the culture supernatant was detected until at least 20 days after the transfection.

Since the infectious virus was recovered from the cells transfected with SHIV3sj-RANTES, cell-free virus stock was prepared, and the kinetics of viral replication and RANTES expression was studied. The growth kinetics of SHIV3sj-RANTES was similar to that of its parental SHIV$3 \mathrm{sj}$. RANTES was produced along with the replication of SHIV3sj-RANTES, but not with SHIV-3sj. The maximum level of RANTES was $47.4 \mathrm{ng} / \mathrm{m} l$ in the culture supernatant of M8166 cells infected with SHIV3sj-RANTES (Fig. 2).

The relationship among genes inserted to SHIV-3sj, length of the ORF of inserted gene, ability of viral replication, and expression of the protein is summarized in Table 1. As shown, the short insert including RANTES (276 bp) and IL-5 (399 bp) resulted in the successful expression from SHIV-3sj, while the construct having longer genes including IL-2 (462 bp), IL-6 (639 bp) and IL-12p35 (762 bp) failed to become replication competent. These results suggest that the length of the insert is an important factor for the replication ability of SHIV-3sj vector. However, we cannot exclude the possibility that specific sequences in IL-2, IL-6 and IL-12p35 genes are harmful for the replication ability of SHIV-3sj vector.

$V p r$ vector of HIV-1 NL-43 was previously developed by Jamieson and Zack to study the pathogenesis of HIV-1 in SCID-hu mice. Two reporter genes, HSAL (402 bp) and HSAS (267 bp) were inserted into the vector. They reported that longer insert resulted in the delay of viral replication [5].

In this study we constructed SHIV vpr vector, and successful expression of the inserted gene up to 399 bp was confirmed. Further study is necessary to make vector accept longer insert. We hope this SHIV vpr vector will contribute to study the pathogenesis of AIDS and development of vaccine.

ACKNOWLEDGEMENT. We are grateful to Dr. Alan Krensky at Stanford University for providing full length RANTES cDNA.

\section{REFERENCES}

1. Gibbs, J. S., Lackner, A. A., Lang, S. M., Simon, M. A., Sehgal, P. K., Daniel, M. D. and Desrosiers, R. C. 1995. J. Virol. 69: $2378-2383$.

2. Haga, T., Kuwata, T., Kozyrev, I., Kwofie, T. B., Hayami, M. and Miura, T. 2000. AIDS Res. Hum. Retroviruses 16: 577582.

3. Haga, T., Kuwata, T., Ui, M., Igarashi, T., Miyazaki, Y. and Hayami, M. 1998. Microbiol. Immunol. 42: 245-251.

4. Haga, T., Shimizu, Y., Okoba, M., Kumabe, S., Goto, Y., Shinjo, T., Ichimura, H., Kuwata, T., Hayami, M. and Miura, T. 2002. Microbiol. Immunol. 46: 849-855.

5. Jamieson, B. D. and Zack, J. A. 1998. J. Virol. 72: 6520-6526.

6. Kestler, H. W., 3rd, Ringler, D. J., Mori, K., Panicali, D. L., Sehgal, P. K., Daniel, M. D. and Desrosiers, R. C. 1991. Cell 65: 651-662.

7. Kozyrev, I. L., Miura, T., Haga, T., Kuwata, T. and Hayami, M. 2001. Arch. Virol. 146: 1051-1062.

8. Kuwata, T., Igarashi, T., Ido, E., Jin, M., Mizuno, A., Chen, J. and Hayami, M. 1995. J. Gen. Virol. 76: 2181-2191.

9. Kuwata, T., Miura, T., Haga, T., Kozyrev, I. and Hayami, M. 2000. AIDS Res. Hum. Retroviruses 16: 465-470.

10. Schall, T. J., Jongstra, J., Dyer, B. J., Jorgensen, J., Clayberger, M., Davis, M. and Krensky, A. M. 1988. J. Immunol. 141: 1018-1025.

12. Takai, T. and Ohmori, H. 1990. Biochim. Biophys. Acta 1048: 105-109. 
13. Tristem, M., Marshall, C., Karpas, A. and Hill, F. 1992. Embo J. 11: 3405-3412.

14. Willey, R. L., Smith, D. H., Lasky, L. A., Theodore, T. S., Earl,
P. L., Moss, B., Capon, D. J. and Martin, M. A. 1988. J. Virol. 62: 139-147. 